\title{
Study of Liapunov Exponents and the Reversibility of Molecular Dynamics Algorithms
}

\author{
Karl Jansen ${ }^{\mathrm{a}}$ and Chuan Liu ${ }^{\mathrm{a} *}$

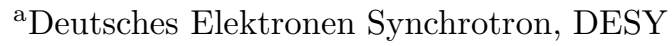 \\ Notkestrasse 85, D-22603 Hamburg, Germany
}

We study the question of lack of reversibility and the chaotic nature of the equations of motion in numerical simulations of lattice QCD.

\section{Introduction}

Molecular dynamics algorithms like the Hybrid Monte Carlo [1] and the Kramers equation [2, 3] algorithm have been playing a major role in numerical simulations of QCD on a Euclidean lattice. In order for these algorithms to fulfill the detailed balance condition, the classical motion governed by the set of Hamilton's equations for the system should be reversible. However, as noticed some time ago [- $[$, this reversibility condition is violated due to the round-off errors in the numerical integration of the equations of motion. Recently, it was pointed out [3] that, due to the chaotic nature of the equations of motion, the round-off errors in the integration which violate the reversibility condition get magnified exponentially with a positive Liapunov exponent $\nu$.

We will focus our discussion on the equations that arise in the simulations of Wilson QCD with two flavors of quarks with degenerate masses. Periodic boundary conditions have been taken for all the fields. The full partition function for Wilson QCD is given by,

$\mathcal{Z}=\int \mathcal{D} U \mathcal{D} \phi^{\dagger} \mathcal{D} \phi \exp \left(-S_{g}-\phi^{\dagger} Q^{-2}[U] \phi\right)$

with $S_{g}$ being the Wilson plaquette action. The fermion matrix $Q[U]$ is a hermitian sparse matrix.

In molecular dynamics algorithms [1],5], one introduces the Hamiltonian:

$\mathcal{H}=\sum_{x, \mu} \frac{1}{2} \operatorname{Tr}\left(H_{x, \mu}^{2}\right)+S_{e f f}\left(U_{x, \mu}, \phi^{\dagger}, \phi\right)$,

\footnotetext{
${ }^{*}$ Speaker at the conference.
}

where $H_{x, \mu}$ is the Gaussian distributed momentum conjugate to the gauge field $U_{x, \mu}$ and takes values in $s u(3)$, the Lie algebra of $S U(3)$. We have also introduced the effective action $S_{\text {eff }}=$ $S_{g}+\phi^{\dagger} Q^{-2} \phi$. Then the gauge fields and its corresponding momenta are updated according to Hamilton's equations of motion:

$\dot{U}_{x, \mu}=i H_{x, \mu} U_{x, \mu}, i \dot{H}_{x, \mu}=\left[U_{x, \mu} F_{x, \mu}\right]_{T . A .}$,

where the symbol $[\cdots]_{T . A}$. stands for taking the traceless antihermitian part of the matrix [5] and the quantity $U_{x, \mu} F_{x, \mu}$ is the total force associated with the link $U_{x, \mu}$. The dot on a field variable indicates the derivative with respect to Monte Carlo time. Eq. (3) defines a Hamilton flow in a phase space manifold which is a direct product of $4 L^{3} T$ factors of $S U(3)$ and $s u(3)$.

\section{Liapunov Exponents}

For any flow described by a set of first-order autonomous differential equations, the concept of Liapunov exponents [6.7] could be introduced locally at each point in the phase space manifold. They describe the mean exponential rate of divergence of two nearby trajectories.

One is thus led to study the time evolution of tangent vectors. We take another differential of eq. (3) and denote $d H_{x, \mu}$ and $d X_{x, \mu}=$ $-i U_{x, \mu}^{-1} d U_{x, \mu}$ as the corresponding tangent vectors, we have:

$$
\begin{aligned}
\dot{d X_{x, \mu}} & =U_{x, \mu}^{-1} d H_{x, \mu} U_{x, \mu}, \\
i d \dot{H}_{x, \mu} & =d\left[U_{x, \mu} F_{x, \mu}\right]_{T . A .} .
\end{aligned}
$$


We introduce the norm in tangent space as:

$D^{2}(\tau)=\sum_{x, \mu} \operatorname{tr}\left(d H_{x, \mu}^{2}(\tau)+d X_{x, \mu}^{2}(\tau)\right)$.

The Liapunov exponent can be defined as:

$\nu=\lim _{D(0) \rightarrow 0} \lim _{\tau \rightarrow \infty} \frac{1}{\tau} \log \frac{D(\tau)}{D(0)}$.

The exponent with the largest real part is called the leading Liapunov exponent.

The numerical calculation of Liapunov exponents of a given flow can be done straightforwardly [6]. Starting at $\tau=0$, we have some initial value of $D^{2}(0)=\sum_{x, \mu} \operatorname{tr}\left(d H_{x, \mu}^{2}+d X_{x, \mu}^{2}\right)$ for a given initial tangent vector $\left(d H_{x, \mu}, d X_{x, \mu}\right)$. We then integrate one step in time with step size $\delta \tau$ using the leapfrog scheme. Now, we evaluate the norm $D(\delta \tau)$ of the new tangent vector and store this information. Next, we rescale the new tangent vector such that its norm is still equal to $D(0)$. Repeating this for $N_{m d}$ steps, we get a sequence of values for the norm: $D(0), D(\delta \tau), \cdots D\left(N_{m d} \tau\right)$. It can be shown [6] that the average

$\nu_{n}=\frac{1}{n \delta \tau} \sum_{k=1}^{n} \log \frac{D(k \delta \tau)}{D(0)}$

is approaching the leading Liapunov exponent when $n \rightarrow \infty$. Its value is independent of the value of $\delta \tau$, as long as $\delta \tau$ is not too large.

\section{Liapunov Exponents for Simulations of QCD}

We have studied the leading Liapunov exponents for various values of $\beta$ and $\kappa$ on $4^{4}$ lattices. In Fig. 1 (a), we plot the exponents as a function of $\beta$ for the pure $S U(3)$ gauge theory. \& We see from this figure that the Liapunov exponents show a significant $\beta$-dependence. It has been conjectured recently [8] that this dependence might be related to the correlation length of the theory.

${ }^{2}$ Similar studies have also been done by the authors of ref. 8. Complete consistent values of the exponent up to $\beta=10$ have been obtained. Note that there is a factor of $\sqrt{2}$ difference between their normalization of Monte Carlo time and ours.

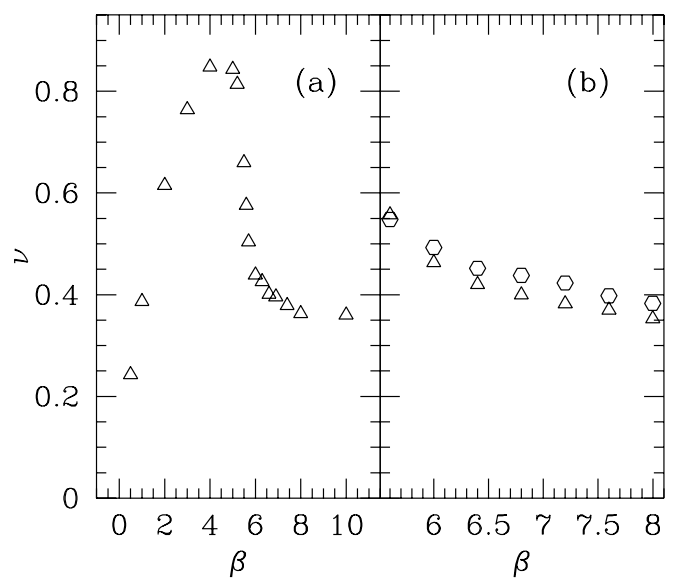

Figure 1. The average leading Liapunov exponents for pure $S U(3)$ gauge theory (a) and full QCD (b) as a function of the gauge coupling $\beta$. In (b), exponents for different values of $\kappa$ are represented by different symbols. The triangles correspond to $\kappa=0.13$ and the hexagons correspond to $\kappa=0.16$.

However, this hypothesis is subject to further investigation.

We have also performed similar investigations of the Liapunov exponents for full QCD with dynamical fermions in the parameter range $5.6 \leq$ $\beta \leq 8.0$ and $0.13 \leq \kappa \leq 1.6$. In Fig. 11(b), we show the final result of the exponents as a function of $\beta$ and $\kappa$. For each value of $\beta$, we have taken 4 different values of $\kappa$, namely $0.13,0.14,0.15$ and 0.16 . Due to their weak dependence on $\kappa$, we only show the exponents for two values of $\kappa$, i.e. $\kappa=0.13$ (triangles) and $\kappa=0.16$ (hexagons). The errors of the points are about the size of the symbols. In the parameter range that we have studied, the Liapunov exponent is roughly $0.4-0.5$.

\section{Consequences of Irreversibility}

Due to rounding errors in the numerical integration, the reversibility condition that is exploited in the proof of the detailed balance con- 
dition for molecular dynamics algorithms is violated [4,3,9,10. When the trajectory length $\tau$ is increasing, we expect the strength of this violation to grow for two reasons. The first one is due to the accumulation of rounding errors, which is expected to grow like $\sqrt{\tau}$. The second cause is due to the chaotic nature of the equations of motion which amplifies the reversibility violation like $\exp (\nu \tau)$ with a positive Liapunov exponent $\nu$.

In order to quantify the effect of these violations in a real simulation, we have run two versions of HMC simultaneously for Wilson QCD with gauge group $S U(2)$ on various lattice volumes $V$. One of them is with 32-bit arithmetic but with 64-bit arithmetic scalar products and summations over the lattice, which mimics the typical situation in simulations on a 32-bit machine. The other one runs with complete 64-bit arithmetic and serves as a reference point for an "exact" program.

The Metropolis step at the end of the trajectory in molecular dynamics algorithms depends on the value of $\Delta H$, the difference of the Hamiltonian after and before the integration of eq. (3). We have compared the two measurements of $\Delta H$, one obtained from the 32-bit arithmetic version and the other from the 64-bit version of the program. 3 The absolute value of the difference between the two values of $\Delta H$ is measured after each molecular dynamics step $\left(<\left|\delta(\Delta H)_{\text {step }}\right|>\right)$, and at the end of the trajectory $\left(<\left|\delta(\Delta H)_{\text {traj }}\right|>\right)$, with the finding that $<\left|\delta(\Delta H)_{t r a j}\right|>$ to be larger than $<\left|\delta(\Delta H)_{\text {step }}\right|>$. We also observe that the value of $<\left|\delta(\Delta H)_{\text {step }}\right|>$ is increasing linearly with $\sqrt{V}=L^{2}$ while the value of $<\left|\delta(\Delta H)_{\text {traj }}\right|>$ seems to grow faster. On $8^{4}, 12^{4}$ and $16^{4}$ lattices, we find $<\left|\delta(\Delta H)_{t r a j}\right|>$ to be $0.08 \%$, $0.18 \%$ and $0.7 \%$ of $\langle|\Delta H|>$ respectively. If this trend is still maintained, at about a $32^{4}$ size lattice, $<\left|\delta(\Delta H)_{t r a j}\right|>$ could reach about $5 \%$ of $\langle|\Delta H|>$ for long trajectories in HMC algorithm, which we think is already dangerous. However, it seems that the quantity $<\left|\delta(\Delta H)_{\text {step }}\right|>$ will remain reasonably small, at about $1 \%$ of $\Delta H$ even on a $32^{4}$ lattice. This suggests that running

\footnotetext{
${ }^{3}$ Other physical observables could also be measured and
} compared and we will report this in the near future. the Kramers equation algorithm will still be feasible on such lattices with 32 -bit arithmetic.

\section{Conclusions}

We have investigated several questions related to the reversibility problem of the molecular dynamics algorithms for the simulation of lattice QCD. We have determined the leading Liapunov exponents for both pure $S U(3)$ gauge theory and full QCD for various bare parameters. We estimated that the effects of rounding errors would become dangerous when running the HMC algorithm on a large lattice of $O\left(32^{4}\right)$ with only 32 -bit precision. In contrast, the comparably performing Kramers equation algorithm has substantially reduced rounding error effects and its use on such lattices is safer.

\section{Acknowledgements}

We thank members of the SESAM collaboration for useful discussions. Critical comments by I. Horváth and A. D. Kennedy are gratefully acknowledged.

\section{REFERENCES}

1. S. Duane, A.D. Kennedy, B.J. Pendleton and D. Roweth, Phys. Lett. B195 (1987) 216.

2. A. M. Horowitz, Phys. Lett. 268B (1991) 247.

3. K. Jansen and C. Liu, Nucl. Phys. B 453 (1995) 375, Nucl. Phys. B 459 (1996) 437.

4. R. Edwards and A.D. Kennedy, private communications.

5. S. Gottlieb et. al., Phys. Rev. D 35 (1987) 2531.

6. G. Benettin, L. Galgani and J. M. Strelcyn, Phys. Rev. A14 2338 (1976).

7. A. J. Lichtenberg and M. A. Lieberman, Regular and Chaotic Dynamics, 2nd ed., Springer-Verlag, 1992.

8. R. Edwards et. al., SCRI preprint, FSUSCRI-96-49, hep-lat/9606004.

9. U. Glässner et. al., hep-lat/9510001.

10. R. C. Brower et. al., Boston university preprint, BU-CCS-950602, hep-lat/9509012. 